\title{
Anti- $\alpha v \beta 3$ antibody guided three-step pretargeting approach using magnetoliposomes for molecular magnetic resonance imaging of breast cancer angiogenesis
}

\author{
This article was published in the following Dove Press journal: \\ International Journal of Nanomedicine \\ 10 January 2013 \\ Number of times this article has been viewed
}

\author{
Chenggong Yan' \\ Yuankui Wu' \\ Jie Feng' \\ Wufan Chen ${ }^{2}$ \\ Xiang Liu' \\ Peng Hao' \\ Ruimeng Yang ${ }^{3}$ \\ Juan Zhang ${ }^{2}$ \\ Bingquan Lin' \\ Yikai Xu' \\ Ruiyuan $\mathrm{Liu}^{4}$ \\ 'Department of Medical Imaging Center, \\ Nanfang Hospital, Southern Medical \\ University, Guangzhou, People's Republic \\ of China; ${ }^{2}$ School of Biomedical Engineering, \\ Southern Medical University, Guangzhou, \\ People's Republic of China; ${ }^{3}$ Department \\ of Radiology, the First Municipal People's \\ Hospital of Guangzhou, Guangzhou, \\ People's Republic of China; ${ }^{4}$ School of \\ Pharmaceutical Science, Southern Medical \\ University, People's Republic of China
}

Correspondence: Yikai Xu

Department of Medical Imaging Center,

Nanfang Hospital, Southern Medical University,

No 1838 Guangzhou Avenue North,

Guangzhou 510515, Guangdong,

People's Republic of China

Tel +86 2062787333

Fax +86 2062787333

Email yikaixu917@gmail.com

Ruiyuan Liu

School of Pharmaceutical Science,

Southern Medical University,

No 1838 Guangzhou Avenue North,

Guangzhou 510515, Guangdong,

People's Republic of China

Tel +862061648196

Fax +862061648196

Email ruiyliu@gmail.com
Purpose: Pretargeting of biomarkers with nanoparticles in molecular imaging is promising to improve diagnostic specificity and realize signal amplification, but data regarding its targeting potential in magnetic resonance (MR) imaging are limited. The purpose of this study was to evaluate the tumor angiogenesis targeting efficacy of the anti- $\alpha v \beta 3$ antibody guided three-step pretargeting approach with magnetoliposomes.

Methods: Polyethylene glycol-modified and superparamagnetic iron oxide-encapsulated magnetoliposomes with and without biotin were synthesized and characterized. The cytotoxicity of both probes was evaluated using the methyl thiazdyl tetrazolium assay, and their cellular uptake by mouse macrophage was visualized using Prussian blue staining. Three-step pretargeting MR imaging was performed on MDA-MB-435S breast cancer-bearing mice by intravenous administration of biotinylated anti- $\alpha v \beta 3$ monoclonal antibodies (first step), followed by avidin and streptavidin (second step), and by biotinylated magnetoliposomes or magnetoliposomes in the targeted or nontargeted group, respectively (third step). The specificity of $\alpha v \beta 3$ targeting was assessed by histologic examinations.

Results: The developed magnetoliposomes were superparamagnetic and biocompatible as confirmed by cell toxicity assay. The liposomal bilayer and polyethylene glycol modification protected $\mathrm{Fe}_{3} \mathrm{O}_{4}$ cores from uptake by macrophage cells. MR imaging by three-step pretargeting resulted in a greater signal enhancement along the tumor periphery, occupying $7.0 \%$ of the tumor area, compared with $2.0 \%$ enhancement of the nontargeted group $(P<0.05)$. Histologic analysis demonstrated the targeted magnetoliposomes colocalized with neovasculature, which was responsible for the MR signal decrease.

Conclusion: These results indicate that our strategy for MR imaging of $\alpha v \beta 3$-integrin is an effective means for sensitive detection of tumor angiogenesis, and may provide a targetable nanodelivery system for anticancer drugs.

Keywords: pretargeting, contrast agents, superparamagnetic iron oxide nanoparticles, avidin-biotin

\section{Introduction}

Tumor angiogenesis, which exhibits the structure and functional abnormalities of blood vessels, plays an important role in tumor growth and development. ${ }^{1}$ In the progress of angiogenesis, the expression of $\alpha v \beta 3$-integrin is upregulated in activated neovascular endothelial cells and several tumor cells, but not in mature, quiescent endothelial cells and normal cells, which makes $\alpha v \beta 3$-integrin a highly selective biomarker for tumor angiogenesis. $^{2}$ Therefore, molecular imaging for overexpression of integrin $\alpha v \beta 3$ 
would facilitate early detection of cancer, evaluation of tumor progression, and cancer therapeutic response.

Much research has focused on the development of diagnostic nanoprobes targeted to $\alpha v \beta 3$-integrin receptors to image tumor vasculature by single-photon emission computed tomography, ${ }^{3}$ positron emission tomography, ${ }^{4}$ optical imaging, ${ }^{5}$ or ultrasound. ${ }^{6}$ Although these imaging modalities are effective and sensitive, the low spatial resolution restricts the exact localization of the expression sites. Magnetic resonance (MR) imaging has been recognized as a powerful diagnostic technique to provide high spatial resolution and excellent soft-tissue contrast. Nevertheless, MR imaging suffers from its major inadequacy - low sensitivity - thus, contrast agents with high affinity and selectivity are necessary for targeting in MR imaging.

Superparamagnetic iron oxide nanoparticles (SPIONs), which can produce predominant $\mathrm{T} 2$ relaxation effects on $\mathrm{T} 2$ images and especially $\mathrm{T} 2 *$ weighted sequences (negative contrast), have widely been applied to cellular and molecular MR imaging. ${ }^{7,8}$ They can be effective in nanomolar concentrations due to their high susceptibility; ${ }^{9}$ thus, they overcome the disadvantage of the low sensitivity of conventional contrast agents. Bare SPIONs generally have hydrophobic surfaces with a large surface area to volume ratio. They tend to aggregate and quickly be taken up by macrophages and accumulated in the reticuloendothelial system (RES) after injection. ${ }^{10}$ One possible approach to avoid capture by RES is synthesis of new contrast agents coated with biocompatible materials. Liposomes have been extensively used as drug carriers, which can be defined as spherical, selfclosed structures formed by concentric lipid bilayers with an aqueous phase inside. ${ }^{11}$ Liposomes entrapping SPIONs in the aqueous lumen exhibit prolonged circulation times and more favorable pharmacokinetic distribution properties. ${ }^{12}$ However, after being coated with nonmagnetic materials, the relaxivity of the contrast agents is lowered, which results in a decrease of sensitivity.

The pretargeting approach based on the avidin-biotin system, exploiting the high specificity and strong affinity ( $\mathrm{Ka}=10^{-15} \mathrm{~mol} / \mathrm{L}$ ) of avidin (or streptavidin [SA]) for biotin, may provide a strategy to improve the sensitivity and specificity of MR imaging. The feasibility of the three-step procedure has been demonstrated in cancer imaging and radioimmunotherapy. ${ }^{13,14}$ The target-specific biotinylated antibodies are injected first to localize onto tumors, followed by avidin, and finally biotinylated effector molecules are administered to bind to tumor pretargeting antibodies. Also, avidin/SA has four sites which can conjugate with biotin, and the avidin-biotin binding is essentially irreversible; ${ }^{15}$ thus, this approach provides an amplification of the signal from the tumor with high specificity. Nevertheless, there is, to the authors' knowledge, no report so far that has successfully characterized tumor angiogenesis via the MR-imaging strategy.

In the present study, polyethylene glycol (PEG)-modified and SPIONs-based magnetoliposomes (ML) were prepared (Figure 1). The synthesis, characterization, cytotoxicity assay, and cellular uptake by mouse macrophage of $\mathrm{ML}$ are reported. Furthermore, the targeting potential of the antibody-guided three-step pretargeting approach to detect angiogenesis was investigated by MR imaging in vivo and histologic examinations.

\section{Materials and methods Materials}

1,2-Distearoyl-sn-glycero-3-phospho-rac-glycerol, sodium salt (DSPG) was purchased from Lipoid GmbH (Ludwigshafen, Germany). 1,2-distearoylsn-glycero-3-phosphoethanol-amine-N- [biotinyl (polyethylene glycol)2000] (Biotin-PEG2000-DSPE) and 1,2-distearoyl-sn-glycero-3 -phosphoethanolamine-N(methoxy [polyethylene glycol]-2000) (DSPE-PEG-2000) were obtained from Avanti Polar Lipids (Alabaster, AL, USA). Lissamine ${ }^{\mathrm{TM}}$ rhodamine B 1,2-dihexadecanoyl -sn-glycero-3- phosphoethanolamine, triethylammonium salt (Rho-DHPE) was from Life Technologies (Carlsbad, CA, USA). 2-(Tris (hydroxymethyl)methylamino) ethane1-sulphonic acid (TES), methyl thiazdyl tetrazolium (MTT), dimethyl sulfoxide, and paraformaldehyde were from Sigma-Aldrich (St Louis, MO, USA). Cholesterol

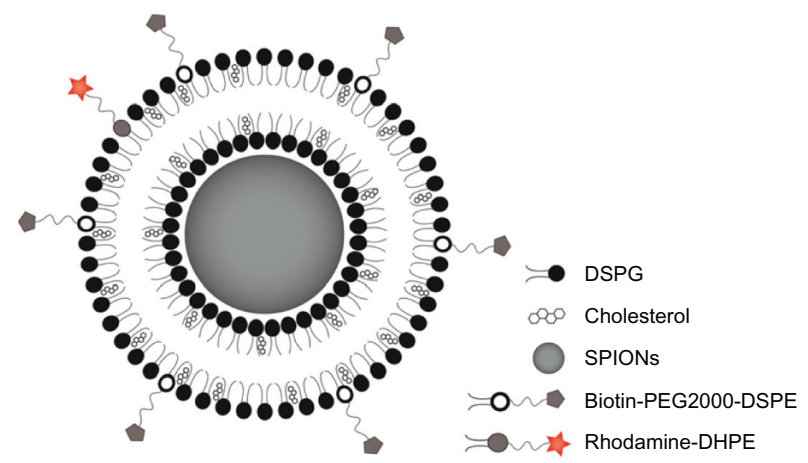

Figure I Schematic representation of a multimodal SPION-based biotinylated magnetoliposome.

Abbreviations: SPIONs, superparamagnetic iron oxide nanoparticles; DSPG, I,2-Distearoyl-sn-glycero-3-phospho-rac-glycerol, sodium salt; Biotin-PEG2000DSPE, I,2-distearoyl-sn-glycero-3-phosphoethanol-amine-N- [biotinyl (polyethylene glycol)2000]; Rhodamine-DHPE, Lissamine ${ }^{T M}$ rhodamine B I,2-dihexadecanoyl-snglycero-3-phosphoethanolamine, triethylammonium salt. 
was purchased from Pubo Chemical Co, Ltd (Guangzhou, China). Streptavidin was obtained from Ruisi Biological Reagents Co, Ltd (Guangzhou, China). Ferric chloride $\left(\mathrm{FeCl}_{3} \cdot 6 \mathrm{H}_{2} \mathrm{O}\right)$, ferrous sulfate $\left(\mathrm{FeSO}_{4} \cdot 7 \mathrm{H}_{2} \mathrm{O}\right)$, concentrated hydrochloric acid $\left(28 \% \mathrm{NH}_{4} \cdot \mathrm{OH}\right.$ in water solution), and lauric acid were purchased from Guangzhou Chemical Reagents Co, Ltd (Guangzhou, China). All other chemicals were of analytical grade.

\section{Preparation of SPIONs}

Synthesis of SPIONs was done according to the literature, ${ }^{16}$ with slight modifications. In general, the synthesis proceeded as follows: appropriate amounts of $\mathrm{FeSO}_{4} \cdot 7 \mathrm{aq}$ (2.78 g) and $\mathrm{FeCl}_{3} \cdot 6 \mathrm{aq}(5.4 \mathrm{~g})$ were mixed at a 1:2 molar ratio and transferred to a three-necked flask, to which $15 \mathrm{~mL}$ of ammonia water was added gradually under vigorous stirring. The reaction was maintained at $60^{\circ} \mathrm{C}$ for 15 minutes to obtain $\mathrm{Fe}_{3} \mathrm{O}_{4}$ nanoparticles (NPs). The black precipitates were isolated by applying a permanent magnet to the $\mathrm{Fe}_{3} \mathrm{O}_{4}$ $\mathrm{NPs}$, then washing with $50 \mathrm{~mL}$ of ammonia/water $(5 / 95, \mathrm{v} / \mathrm{v})$ three times in sequence, then the particles were combined with $1 \mathrm{~g}$ of lauric acid (dissolved in $50 \mathrm{~mL}$ of ammonia/ water $[5 / 95, \mathrm{v} / \mathrm{v}])$, reacted at $90^{\circ} \mathrm{C}$ for several minutes, and finally lauric acid-stabilized SPIONs were obtained.

\section{Preparation of ML}

Liposomes were prepared by a thin lipid film hydration method. DSPG, cholesterol, and biotin-PEG2000-DSPE with a molar ratio of 3:2:0.15 were dissolved in chloroform. Nonbiotinylated control liposomes contained DSPG, cholesterol, and PEG2000-DSPE at a molar ratio of 3:2:0.15. As a fluorescent marker, $0.2 \mathrm{~mol} \%$ of Rho-DHPE was added. The solvent was removed by rotary evaporation, followed by additional drying under nitrogen. The dry lipid film was hydrated in 10 mL TES (5 microM, pH 7.0). Subsequently, the mixture was dispersed by ultrasonic cleaner (200 W, 10 minutes) and then sonicated by ultrasonic disintegrator ( $150 \mathrm{~W}, 30$ minutes). The phospholipid vesicle was purified by centrifugation at $37^{\circ} \mathrm{C}$ for 20 minutes at $2500 \mathrm{~g} /$ minute.

ML and biotinylated ML (Bt-ML) were prepared from lauric acid-stabilized SPIONs $(0.5 \mathrm{~mL}$; Fe concentration $22 \mathrm{mg} / \mathrm{mL}$ ), which were dialyzed (molecular weight cutoff: 10,000$)$ at $37^{\circ} \mathrm{C}$ for 3 days in the presence of phospholipid vesicles ( $10 \mathrm{~mL}$ containing $0.15 \mathrm{mmol}$ phospholipid) with regular changes of the buffer ( 5 microM TES, PH 7.0 ) by a self-assembly procedure to form lipid-coated SPIONs.

\section{Biotinylation of monoclonal antibodies (MoAb)}

Anti- $\alpha v \beta 3$ monoclonal antibodies (Biolegend, San Diego, CA, USA) were biotinylated with Sulfo-NHS-LC-Biotin following the manufacturer's (Pierce, Rockford, IL, USA) protocol. After purification by ultrafiltration with an Amicon Ultra-15 Centrifugal Filter Unit with a $10 \mathrm{kDa}$ membrane from EMD Millipore (Billerica, MA, USA), the final biotin/antibody ratio was $\approx 4$ as determined by the HABA method (Pierce).

\section{Characterization}

The size and morphology of the synthesized NPs were observed using a transmission electron microscope ([TEM] H-7650; HITACHI, Tokyo, Japan) operating at $200 \mathrm{kV}$. ML and Bt-ML were stained with $1 \%$ phosphotungstic acid for negative staining to distinguish their shell from the core, but SPIONs were not. The mean size distribution of the particles was determined using a Malvern Zeta-sizer 3000HS (Malvern Instruments, Malvern, UK) operating at $632.0 \mathrm{~nm}$. X-ray diffraction (XRD) was performed using a XRD system (D-MAX2200 VPC; Rigaku Corporation, Tokyo, Japan) equipped with $\mathrm{Cu} / \mathrm{Ka}$ radiation at a scanning rate of $4^{\circ} /$ minute in a 2 theta range of $10^{\circ} \sim 70^{\circ}$ at $40 \mathrm{kV}$ and $30 \mathrm{~mA}$. The loading content of iron in the particles was determined using an atomic absorption spectrophotometer (Z-5000; HITACHI).

\section{Magnetic property measurements}

The NPs were prepared in $0.01,0.02,0.03,0.04$, and 0.05 microM of Fe concentrations. The T2 of these solutions were determined on a 3.0T MR system (Signa Excite; General Electric, Milwaukee, WI, USA). A T2 mapping sequence (TR: $3000 \mathrm{~ms}$, TE: 20, 40, 60, and $80 \mathrm{~ms}$, matrix $512 \times 160$, field of view $200 \mathrm{~mm}$ ) was used to measure transverse relaxation time. Images of the various solutions were analyzed by defining regions of interest (ROI) in each test tube. Relaxivity ( $\mathrm{r} 2$ ) was calculated through the curve-fitting of T2 $\left(\mathrm{s}^{-1}\right)$ vs the Fe concentration (microM).

A vibration sample magnetometer (MPMS XL-7 magnetometer; Quantum Design, Inc, San Diego, CA, USA) was used to characterize the magnetic properties of the particles. The hysteresis of the magnetization was recorded at $300 \mathrm{~K}$ obtained under a circulate magnetic field ranged between +1 and -1 Tesla.

\section{Cell culture}

All the cell lines were gifts from the Research Center of Clinical Medicine in Nanfang Hospital (Guangzhou, China). 
Both breast cancer cell lines of MDA-MB- 435S ( $\alpha v \beta 3$ integrin positive) and MCF-7( $\alpha v \beta 3$ negative) cells were grown in Dulbecco's modified Eagle's medium, and RAW264.7 in RPMI-1640 supplemented with 10\% fetal bovine serum, penicillin $(100 \mathrm{U} / \mathrm{mL})$, and streptomycin $(100 \mathrm{U} / \mathrm{mL})$ in a humidified incubator at $37^{\circ} \mathrm{C}$ with $5 \% \mathrm{CO}_{2}$ in the air.

\section{Cytotoxicity assay}

In vitro cytotoxicity of the NPs was assessed using the MTT assay of MDA-MB-435S and MCF-7. Briefly, the cells were seeded in a 96-well plate at the density of $4 \times 10^{4}$ cells/well for 48 hours, then the culture medium was replaced with a $200 \mu \mathrm{L}$ medium containing ML or Bt-ML (at 200, 400, $800,1200,1600$, and $2000 \mu \mathrm{M} \mathrm{Fe}$ ) and kept incubated for 20 hours. Subsequently, $50 \mu \mathrm{L}$ of MTT solution $(5 \mathrm{mg} / \mathrm{mL})$ was added to the wells and incubated for 4 hours in a humidified atmosphere prior to the addition of $150 \mu \mathrm{L}$ of dimethyl sulfoxide into each well to dissolve the formazan crystals, and gently shaken for 10 minutes so that complete dissolution was achieved. The reaction mixture on each well of the 96-well culture plate was measured using the enzyme-linked immunosorbent assay reader BIOTEK ELX800 at a wavelength of $570 \mathrm{~nm}$. The control group contained cells and cell culture medium without NPs.

\section{Visualization of the cellular uptake by Prussian blue staining}

RAW264.7 cells were seeded onto the 6-well plate with $5 \times 10^{5}$ cells/well and incubated for 24 hours. Then the supernatant was removed and $2 \mathrm{~mL}$ of the full RPMI-1640 culture medium containing SPIONs, ML, and Bt-ML (Fe concentration of $50 \mu \mathrm{g} / \mathrm{mL}$ ) was added to the RAW264.7 cells, respectively. After incubation at $37^{\circ} \mathrm{C}$ for 2 hours, the supernatant was removed and cells were washed three times with phosphate-buffered saline. Then, the cells were fixed by $2 \mathrm{~mL}$ of $4 \%$ paraformaldehyde solution for 20 minutes. After fixation, the cells were stained with a filtrated and fresh prepared potassium ferrocyanate solution (mixture of equal volume of $4 \%$ potassium ferrocyanate with $4 \%$ hydrochloric acid) for 30 minutes at $37^{\circ} \mathrm{C}$ causing a deep blue complex, rewashed, and then counterstained with nuclear fast red for 5 minutes. The wells without NPs were used as blank.

\section{In vivo $M R$ imaging}

All procedures were approved by the animal center of Southern Medical University (Guangzhou, China). Tumor xenografts with $1 \times 10^{7} \mathrm{MDA}-\mathrm{MB}-435 \mathrm{~S}$ cells were implanted into the right caudal mammary fat pad of female mice (NOD/SCID; Huafukang Company, Beijing, China). Six to 8 weeks after implantation when the tumor reached $0.8-1.2 \mathrm{~cm}$ in diameter, the animals were randomized into two groups: targeted Bt-ML and nontargeted ML group for MR scan ( $\mathrm{n}=5$ per group).

The mice were subjected to injections via the tail vein with $150 \mu \mathrm{g}$ biotinylated anti- $\alpha v \beta 3 \mathrm{MoAb}$ over 2 minutes (first step). After 36 hours, $1 \mathrm{mg}$ of cold avidin (Santa Cruz Biotechnology, Santa Cruz, CA, USA) was injected over 2 minutes, followed by an additional $0.5 \mathrm{mg}$ of cold streptavidin 10 minutes later (second step). Two hours later, ML or Bt-ML was injected via the tail vein at a dose of $80 \mu \mathrm{mol} \mathrm{Fe} /$ $\mathrm{kg}$ (third step). Mice were anesthetized with an intraperitoneal injection of pentobarbital sodium $(60 \mathrm{mg} / \mathrm{kg}) 10$ minutes before the MR studies. MR imaging was performed before and 2 hours post-injection with a 3.0T MR system (Signa Excite) equipped with a mouse-imaging coil. Coronal T2-weighted $\mathrm{SE}(\mathrm{TR} / \mathrm{TE}=4000 / 85 \mathrm{~ms}, \mathrm{FOV}=12 \mathrm{~cm}$, matrix $=320 \times 224$, $\mathrm{NEX}=4$, thickness/interval $=2.5 / 0.2 \mathrm{~mm}$ ).

The pixel intensities from the T2-weighted images were analyzed with MATLAB software (The Math-Works, Natick, MA, USA). ${ }^{17,18}$ In each baseline and 2 hours post-injection T2-weighted image slice, a ROI was manually placed around the tumor edge on each baseline slice, and the standard deviation (SD) of the average tumor signal at baseline was calculated. Subsequently, serial images were spatially coregistered using a cross-correlation routine, and the tumor ROI mask was copied to each time point. At 2 hours post-injection, pixels with an MR signal intensity decrease $\leq 3$ SD below the baseline tumor signal were considered significant. The enhanced fraction within the whole tumor for each individual mouse was determined by the number of enhanced pixels divided by the number of total pixels within the tumor.

\section{Fluorescence immunohistochemistry and Prussian blue staining}

After MR imaging, animals were sacrificed following anesthesia and cervical dislocation. Tumors were resected, and quickly frozen in an optimal cutting temperature compound for immunohistochemistry. Frozen tumor tissue slices ( $5 \mu \mathrm{m}$ thick) were fixed in acetone for 10 minutes, and then dried in the air for 30 minutes. Nonspecific binding of antibodies was blocked by incubation with donkey serum for 30 minutes at room temperature. The sections were incubated overnight with rat anti-mouse CD31 monoclonal antibody (BD Pharmingen, San Jose, CA, USA), then washed and incubated with fluorescein isothiocyanate (FITC) goat anti-rat secondary antibody (Jackson ImmunoResearch, 
West Grove, PA, USA). 4,6'-diamidino-2-phenylindole (Molecular Probes, Eugene, Oregon, USA) was used for cell nuclei staining.

Sections were also stained with Prussian blue according to standard clinical pathology protocols.

\section{Statistical analysis}

Statistical analysis was performed using a two-tailed unpaired Student's $t$-test at the $P<0.05$ level. All data were expressed in the form of mean $\pm \mathrm{SD}$. All tests were performed using SPSS version 13.0 (IBM Corporation, Armonk, NY, USA).

\section{Results}

\section{Characterization of ML}

TEM images (Figure 2) show NPs in a dry state. From the TEM images (Figure 2A), the diameter of spherical SPIONs was estimated to be $13.6 \mathrm{~nm}$. After incorporation of SPIONs with liposomes, an about $4 \mathrm{~nm}$ thick amorphous edge around the SPIONs was observed. We also measured hydrodynamic diameters and distribution of ML using a dynamic light scattering (DLS) instrument. The particle sizes, hydrodynamic diameters, and polydispersity indices are summarized in Table 1. Pure SPIONs had a particle size of $86.9 \mathrm{~nm}$ with a polydispersity index of 0.146 . The incorporation of SPIONs into the liposomes resulted in increased particle size (120 129 nm) due to the formation of coating layers of liposomes. As shown in Table 1, the particle size determined by TEM was smaller compared with the particle size of the same particles obtained from DLS measurements. These results confirm the presence of a liposome hairy layer on the particle surface, which increases the value of the average hydrodynamic diameter during the DLS measurements and which is collapsed onto the particle surface during the drying of the TEM sample. It should also be remembered that while TEM provides the number-average particle size,
DLS gives the zeta-average, which is sensitive to large-size particles.

XRD patterns of the synthesized particles were analyzed to determine the crystal phase of the SPIONs and ML (Figure 3). All of the particles (SPIONs, ML, and Bt-ML) showed diffraction peaks at $30.1^{\circ}, 35.5^{\circ}, 43.1^{\circ}, 53.4^{\circ}, 57.0^{\circ}$, and $62.6^{\circ}$, due to face-centered cubic lattice structures 220 , $311,400,422,440$, and 511 which are characteristic peaks of the $\mathrm{Fe}_{3} \mathrm{O}_{4}$ crystal structure. Additionally, there are no peaks at $31^{\circ}$ corresponding to $\gamma-\mathrm{Fe}_{2} \mathrm{O}_{3}$ and $\alpha-\mathrm{Fe}_{2} \mathrm{O}_{3}$ for 210 and 213 in XRD patterns, supporting the purity of synthesized iron oxide nanoparticles. The results indicated that liposomal encapsulation of the magnetic cores did not make significant changes in the crystal phase of $\mathrm{Fe}_{3} \mathrm{O}_{4}$.

\section{Magnetic property measurements}

The T2 relaxivity of the NPs at 3.0T MR was examined (Figure 4A). The results showed that the MR imaging signal intensity of the NPs decreased with the increase of $\mathrm{Fe}$ concentration. The NPs were well-fitted by a line within the analyzed range of iron concentration (0.01-0.05 microM), thus exhibiting the typical property of SPIONs in shortening $\mathrm{T} 2$ relaxation time. The specific relaxivity values of SPIONs, ML, and Bt-ML were found to be $0.722 \times 10^{3}, 0.611 \times 10^{3}$, and $0.675 \times 10^{3}$ microM $^{-1} \mathrm{~s}^{-1}$, respectively.

The magnetic property of SPIONs, ML, and Bt-ML was evaluated by vibration sample magnetometer to confirm the feasibility and sensitivity as MR imaging nanoprobes (Figure 4B). Typical hysteresis curves regarding superparamagnetic behaviors were observed. The magnetization decreased from plateau value to zero when magnetic field intensity decreased, which implied the NPs would respond well to magnetic fields without any permanent magnetization. The specific saturation magnetism $\sigma_{\mathrm{s}}$ at $1 \mathrm{~T}$ for ML and Bt-ML were $11.7 \mathrm{emu} / \mathrm{g}$ and $8.05 \mathrm{emu} / \mathrm{g}$, which were smaller than the value for the SPIONs (41.2 emu/g). The decrease of the
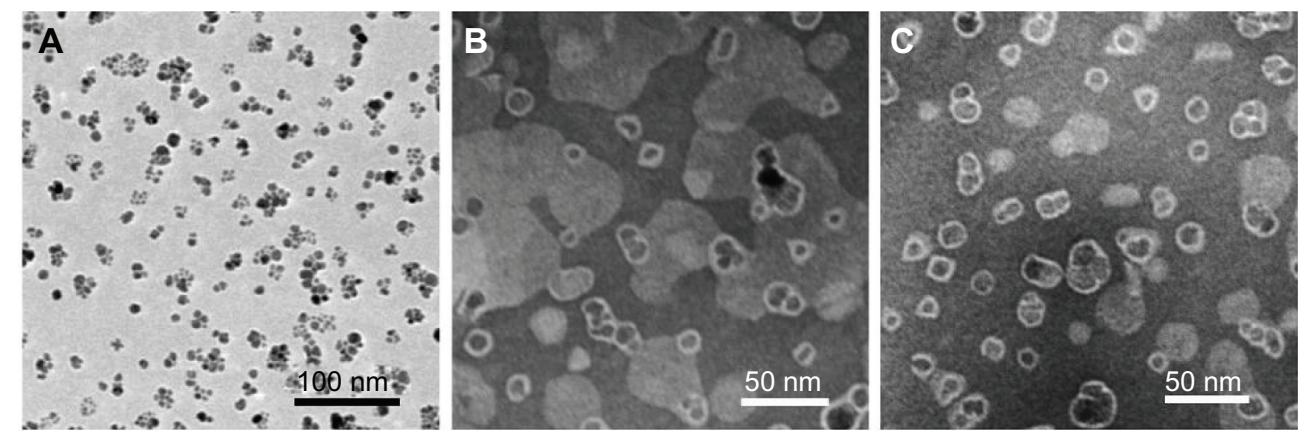

Figure 2 Transmission electron microscopy images of (A) SPIONs, (B) ML, and (C) Bt-ML.

Abbreviations: SPIONs, superparamagnetic iron oxide nanoparticles; ML, magnetoliposomes; Bt-ML, biotinylated magnetoliposomes. 
Table I Physical characteristics of nanoparticles

\begin{tabular}{llll}
\hline & $\begin{array}{l}\text { Particle size } \\
(\mathbf{n m})\end{array}$ & $\begin{array}{l}\text { Hydrodynamic } \\
\text { diameter } \\
(\mathbf{n m})\end{array}$ & $\begin{array}{l}\text { Polydispersity } \\
\text { index }\end{array}$ \\
\hline SPIONs & $13.6 \pm 2.9$ & 86.9 & 0.146 \\
ML & $17.0 \pm 2.4$ & 129.0 & 0.186 \\
Bt-ML & $17.6 \pm 3.2$ & 120.0 & 0.235 \\
\hline
\end{tabular}

Abbreviations: SPIONs, superparamagnetic iron oxide nanoparticles; ML, magnetoliposomes; Bt-ML, biotinylated magnetoliposomes.

magnetization of such coated particles might be due to the coating of nonmagnetic liposomes on the magnetic nanoparticles and/or the decrease in their iron oxide content.

\section{Cytotoxicity assay}

In order to investigate the cytotoxicity of the novel nanoparticles carrier, both MDA-MB-435S and MCF-7 were incubated with ML and Bt-ML, and the cytotoxicity was assessed using MTT assay. Cell viability was apparently unaltered in the entire test dosage range from 200 to $2000 \mu \mathrm{M}$ Fe as depicted in Figure 5. This result indicated that ML and Bt-ML are biocompatible and low-toxic at the given $\mathrm{Fe}$ concentration range $(200-2000 \mu \mathrm{M})$.

\section{NP uptake by macrophages}

In order to evaluate the capacity of ML to evade capture by macrophage cells in vitro, the cellular uptake of NPs in RAW264.7 cells was visualized by Prussian blue staining (Figure 6). Compared with the control group without contrast agents (Figure 6A), RAW264.7 cells incubated with SPIONs were stained in intensive blue color under $50 \mu \mathrm{g} / \mathrm{mL}$ of the $\mathrm{Fe}$ concentration in culture medium. Blue areas or spots could

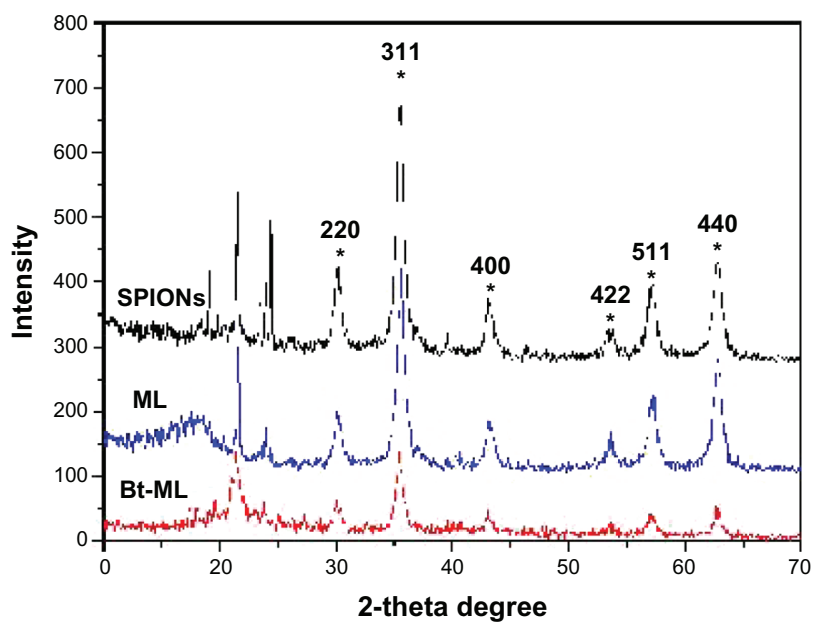

Figure 3 X-ray diffraction pattern of SPIONs, ML, and Bt-ML.

Abbreviations: SPIONs, superparamagnetic iron oxide nanoparticles; ML, magnetoliposomes; Bt-ML, biotinylated magnetoliposomes. be seen in almost every cell (Figure 6B). In comparison, the RAW264.7 cells incubated with ML or Bt-ML showed a little weak blue color appearance (Figure 6C and D). The result indicated that SPION entrapment into liposomes and modification by PEG could dramatically decrease the internalization of the NPs by macrophage cells, which allows more contrast agents access to the target vasculature.

\section{In vivo $M R$ imaging}

The in vivo MR imaging was performed before injection and 2 hours post-injection (Figure 7A). The pre-contrast images also contained some enhanced pixels, as their signal intensities were below the threshold. For mice receiving Bt-ML, the spatial distribution of enhanced pixels was heterogeneous and mainly in the periphery of the tumor. For mice receiving ML, pixel signal enhancement was much less pronounced and scattered within the tumor rim. The enhanced fraction in tumors was $7.0 \% \pm 1.8 \%(P<0.05)$ at 2 hours post-injection of targeted Bt-ML, while only a $2.0 \% \pm 1.1 \%$ decrease was observed in the nontargeted group (Figure 7B). Additionally, we found high signal reductions in the liver at 2 hours postinjection of Bt-ML and ML, respectively.

\section{Fluorescence immunohistochemistry and Prussian blue staining}

Ex vivo fluorescence microscopy was used to investigate the binding location of NPs inside tumor tissues. Rhodamine fluorescence from $\alpha v \beta 3$-targeted Bt-ML was colocalized with the angiogenic endothelial cells within tumor tissues, and mainly distributed to the periphery of the tumor (Figure 8A-C). Little fluorescence was also observed outside the vessel wall or lumen. In contrast, fluorescence from ML was only found in the interstitial space of the tumor but not colocalized with endothelial cells (Figure 8D-F).

To further verify the accumulation of the SPION encapsulated NPs in the tumor tissue, Prussian blue staining was carried out as shown in Figure 9. In good agreement with fluorescence immunohistochemistry, blue spots of Bt-ML showed vascular localization. In the nontargeted group, only a little ML was observed with an unspecific distribution. The neovascular spatial distribution seen microscopically, was consistent with the contrast enhancement patterns of angiogenesis observed in MR imaging.

\section{Discussion}

In the present study, we demonstrated that the antibody-guided three-step pretargeting approach was highly effective for molecular imaging of angiogenesis in vivo. SPION-based ML 

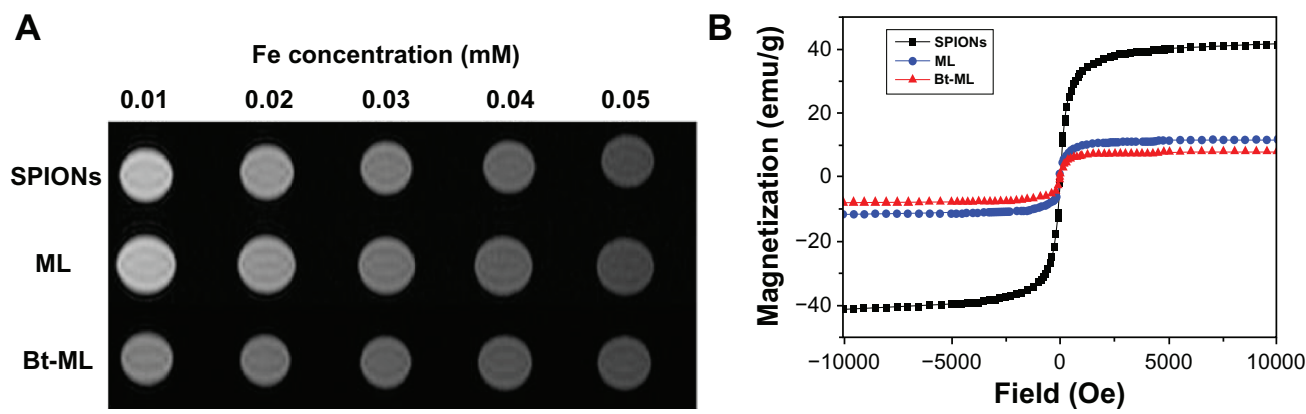

Figure 4 Magnetic property measurements of nanoparticles: (A) T2-weighted MR images and (B) magnetic behavior of SPIONs, ML, and Bt-ML. Abbreviations: MR, magnetic resonance; SPIONs, superparamagnetic iron oxide nanoparticles; ML, magnetoliposomes; Bt-ML, biotinylated magnetoliposomes.

are sensitive and biocompatible contrast agents for detection of $\alpha v \beta 3$-integrin overexpressing on angiogenic endothelium. MR signal enhancement revealed the peripheral spatial distribution of neovasculature, which was highly correlated to the histologic characteristics of tumors.

To enable targeted imaging of tumor angiogenesis, highly efficient NPs coated with biocompatible polymers are desirable. ${ }^{19} \mathrm{ML}$, with a high payload of NPs and biocompatibility, have been studied as bone marrow MR contrast agents. ${ }^{20}$ In the present study, we prepared SPION-encapsulated ML with superparamagnetism and high molar relaxivity. Our results demonstrated that even at the Fe concentration of $2000 \mu \mathrm{M}$, the cell viability evaluated by the MTT assay still remained above $75 \%$, which is important for further biological applications. Besides, after liposomal coating, the capture of NPs by mouse macrophage cells was significantly reduced. Incorporation of PEG into lipid bilayers not only protects against possible unwanted interactions with plasma protein or neighboring cells, ${ }^{21}$ but also can be functionalized with a distal moiety to target biomarkers, ${ }^{22}$ consequently allowing more ML to accumulate at the targeted sites.

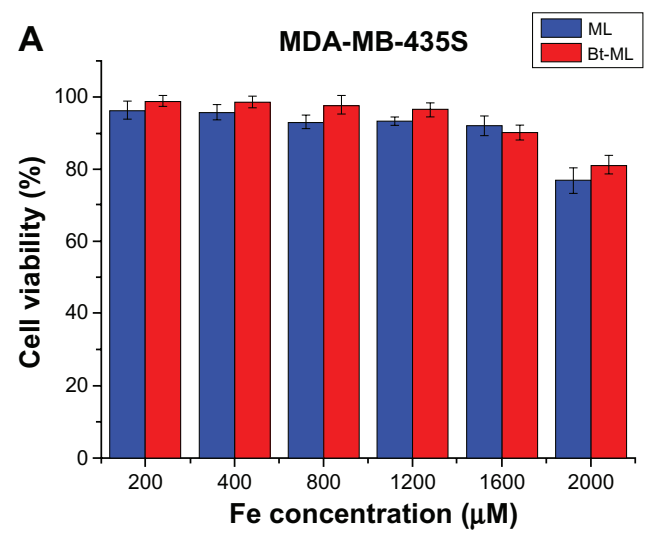

To specifically target the desired biomarkers of tumors, contrast agents can potentially be conjugated with many varieties of ligands including antibodies, ${ }^{23-25}$ peptides, ${ }^{26}$ and polysaccharides. ${ }^{27}$ For example, antibody-conjugated paramagnetic liposomes have been used to target $\alpha v \beta 3$-integrin for MR imaging-based visualization of angiogenesis. ${ }^{23}$ However, antibodies will considerably increase the already large size of contrast agents, resulting in unfavorable biodistribution. ${ }^{25}$

Compared to a directly labeled MoAb approach, an antibody-guided pretargeting approach, through a stable bridge of biotin-avidin, holds promise in improving the tumor/nontumor ratio. ${ }^{28}$ Several pretargeting protocols using different multistep avidin-biotin methods have been proposed. ${ }^{14,29,30}$ A method including MoAb-biotin/avidin/ biotin described by Paganelli et al ${ }^{14}$ obtained high selective tumor uptake of radionuclide with less systemic toxicity than covalent conjugates of MoAb. The three steps of antibodyguided pretargeting are as follows: (1) the localization of biotinylated monoclonal antibodies on targeted cells; (2) avidination of the pretargeted biotinylated antibodies and clearance of circulating biotinylated antibodies by an

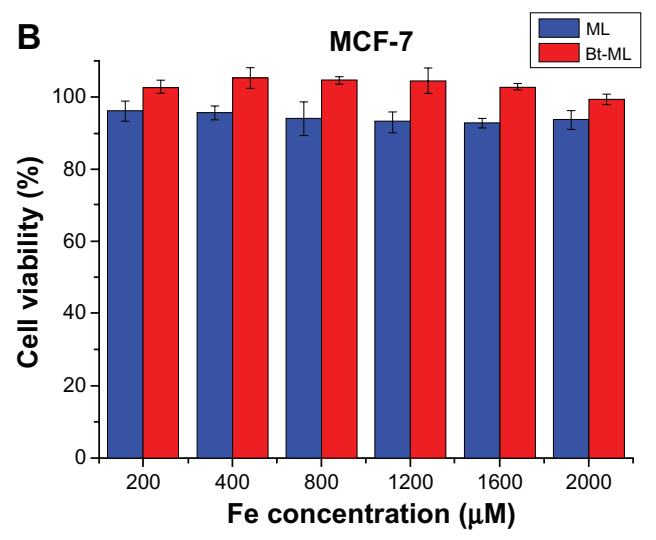

Figure 5 In vitro cell viability of (A) MDA-MB-435S and (B) MCF-7 cells incubated with ML and Bt-ML with different iron concentrations determined by MTT assay. Abbreviations: MDA-MB-435S, human breast cancer cells; MCF-7, hormone dependent breast cancer cells; ML, magnetoliposomes; Bt-ML, biotinylated magnetoliposomes; MTT, methyl thiazdyl tetrazolium. 

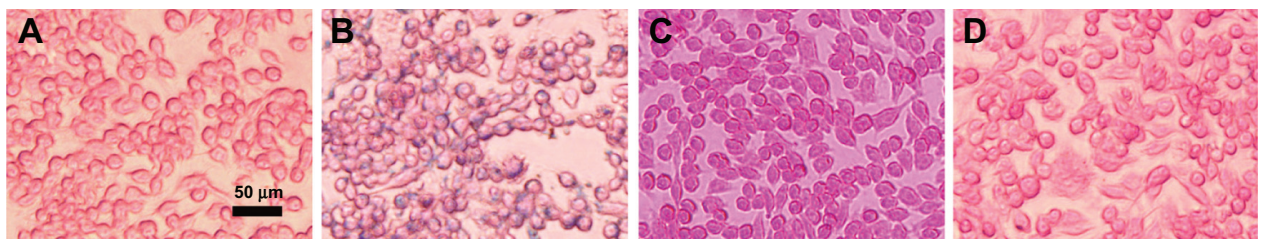

Figure 6 Prussian blue staining micrographs of macrophage cells (RAW264.7). (A) Control without contrast agent, and cells treated with (B) SPIONs, (C) ML, and (D) Bt-ML. Note: Blue stain density reflects the level of SPIONs accumulation within cells.

Abbreviations: SPIONs, superparamagnetic iron oxide nanoparticles; ML, magnetoliposomes; Bt-ML, biotinylated magnetoliposomes.

excess of cold avidin/SA; (3) postlabeling of the target by biotinylated contrast agents. ${ }^{29}$ To exploit the targeted receptor-ligand mechanism for delivering SPION-based ML to the tumor region, the antibody-guided three-step pretargeting approach was used in our study (Figure 7C). Through the linkage of pretargeting biotinylated MoAb and avidin/
SA, Bt-ML rapidly bound to target $\alpha v \beta 3$-integrin with high specificity, resulting in a heterogeneous and more pronounced signal decrease in the rim of tumors compared to the nontargeted group. The signal was amplified for the reason that more than one molecule of avidin/SA can bind to a single biotinylated MoAb molecule localized on the tumor and that
A
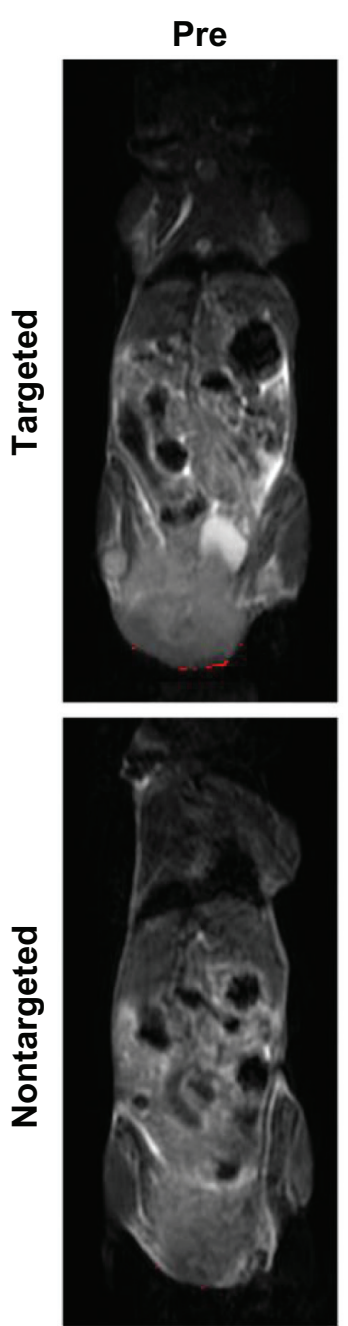

Post
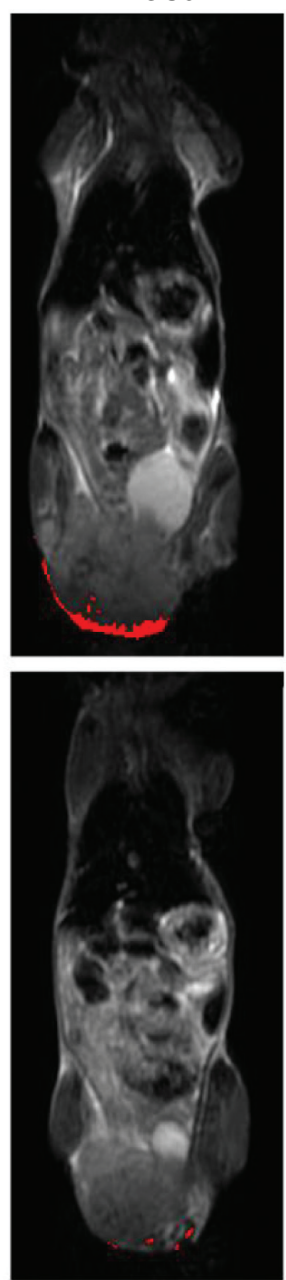

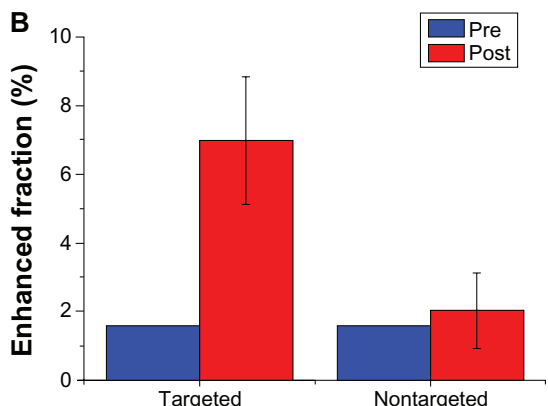

C

Targeted

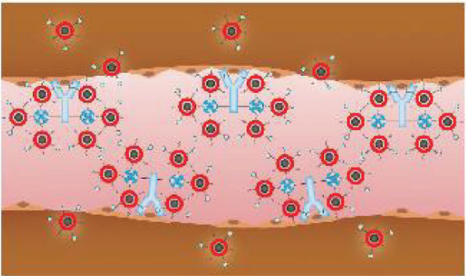

Nontargeted

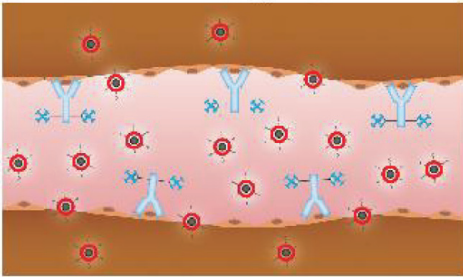

Step 1 Biotinylated MoAb

Step 2 Avidin/streptavidin $\$$

Step 3 Bt-ML or ML

Figure $7 \mathrm{MR}$ detection of the tumors via the anti- $\alpha v \beta 3$ antibody guided three-step pretargeting approach. (A) T2-weighted MR images of tumor-bearing mice before and 2 hours after injection of targeted Bt-ML and nontargeted ML, respectively. Enhanced pixels within the tumors were color coded in red. (B) Enhanced fraction of the tumor region before and after 2 hours after injection of contrast agents. (C) Schematic representation of the antibody-guided three-step pretargeting approach.

Abbreviations: MR, magnetic resonance; Bt-ML, biotinylated magnetoliposomes; ML, magnetoliposomes. 

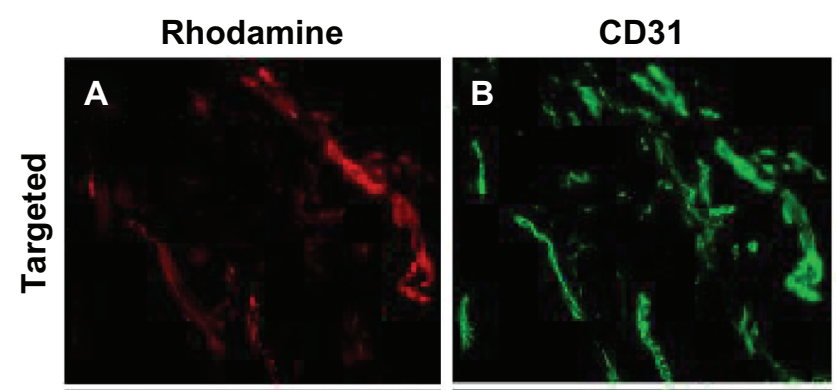

\section{Merged with DAPI}
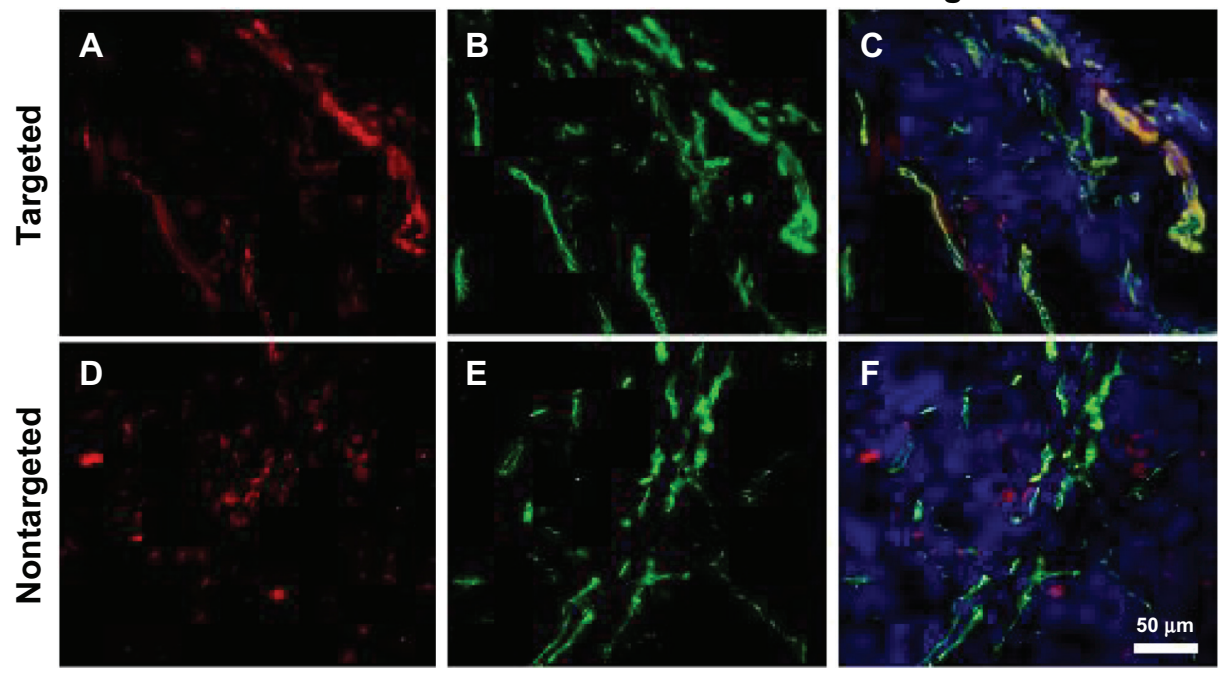

Figure 8 Fluorescence microscopy images of tumor tissues. (A-C) Bt-ML colocalizes with the angiogenic vasculature from the tumor periphery; (D-F) ML is seen beyond the vasculature.

Notes: Liposomal fluorescence from rhodamine (red) is shown in the left panel. Endothelium stained using an antimouse CD3I antibody (green) is shown in the middle panel. Color overlays with nuclei counterstained DAPI (blue) are shown in the right panel.

Abbreviations: DAPI, 4,6'-diamidino-2-phenylindole; Bt-ML, biotinylated magnetoliposomes.

more than one biotinylated effector can bind to an avidin/ SA molecule. Zhu et a ${ }^{30}$ utilized PAMAM dendrimer-based contrast agents to target Her-2/neu receptors by a three-step pretargeting approach for MR imaging, but produced limited selective enhancement. This may be due to the binding sites of avidin being saturated by biotinylated monoclonal antibodies. In our study, the intratumoral distribution of $\alpha v \beta 3$-integrin was sparse and much excessive avidin/SA was administered, so the possibility of saturation of avidin/ SA by biotinylated MoAb was much less. The pronounced MR enhancement in the targeted group further demonstrated the success of our strategy. Additionally, in contrast with our in vitro experiments, strong signal reduction in the liver was observed for ML and Bt-ML at 2 hours post-injection, which indicates that a portion of contrast agents were taken up by RES. Various conditions (such as water dilution or plasma

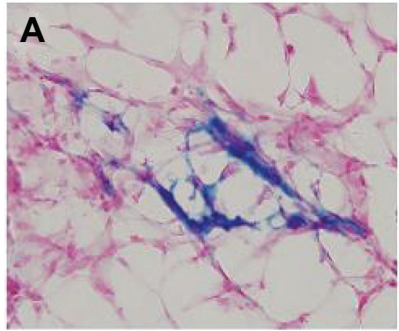

Targeted

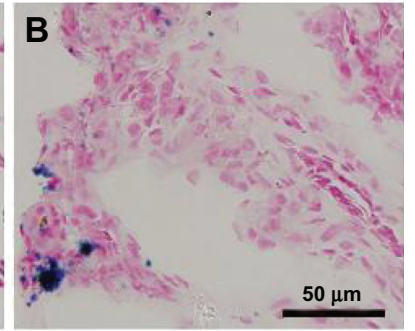

Nontargeted
Figure 9 Prussian blue staining images of tumor tissues from mice after injection of (A) targeted Bt-ML and (B) nontargeted ML.

Abbreviations: Bt-ML, biotinylated magnetoliposomes; ML, magnetoliposomes. buffering) in vivo may affect properties of ML and the situation will be certainly different. ${ }^{31}$

For validation of the MR imaging findings and to establish the exact site and mechanism of accumulation of the NPs, fluorescence microscopy and Prussian blue staining of the excised tumor were performed. Histologic results further demonstrated that through the pretargeting approach, Bt-ML targeted $\alpha v \beta_{3}$-integrin colocalized with angiogenic endothelium of tumors in vivo, in agreement with previous reports. ${ }^{32}$ The selective targeting of Bt-ML to vascular structures is responsible for the MR signal decrease. Besides, a diffuse pattern of fluorescence was also found in the interstitial space within the tumor for both tested NPs, which is most likely due to the enhanced permeability of the neovasculature and retention effect of NPs. These results agree with previous findings that RGD-functionalized bimodal probes enable image angiogenesis by characterization of $\alpha v \beta_{3}$-integrin with a combination of MR imaging and fluorescence microscopy. ${ }^{33}$

It should be noted that long-circulating NPs allow for prolonged time for extravasation from neovasculature with increased permeability. ${ }^{34}$ Because peak concentration of NPs in tumors is $1-3$ hours post-injection, ${ }^{29}$ we performed MR imaging in vivo at 2 hours post-injection to improve the specificity of the tumor imaging. In our study, unspecific contrast enhancement was also observed in nontargeted tumors, but much less pronounced than the targeted group. Compared to specific localization, the nonspecific 
extravasation was significantly less extensive in fluorescent microscopy.

Pretargeting is a useful tool to improve sensitivity and specificity of molecular imaging. This approach may facilitate assessment of angiogenic activity and noninvasive evaluation of chemotherapeutic response. In addition, liposomes may be also used as delivery vehicles to encapsulate therapeutic drugs to avoid severe damage to healthy tissues, which makes target-specific theranostics possible.

\section{Conclusion}

In conclusion, we have prepared SPION-based ML with superparamagnetism, biocompatibility, and low cytotoxicity. Our results demonstrated that with an antibody-guided three-step pretargeting approach, ML were sensitive enough to allow for MR imaging detection of tumor angiogenesis. Additionally, the MR enhancement revealed the predominantly peripheral distribution of angiogenesis, which was highly consistent with the histologic distribution of $\alpha v \beta 3$ targeted NPs. Thus, our strategy with liposomal carriers may provide a versatile platform for assessment of angiogenic activity at the molecular level and can be used as a targetspecific anticancer drug delivery system.

\section{Acknowledgments}

We thank Yingxin Yu (Department of Immunology, Southern Medical University, China) for technical assistance in antibodies biotinylation. This research was supported in whole or in part by the National Natural Science Foundation of China (30870708, 81071146, and 81271642).

\section{Disclosure}

The authors report no conflicts of interest in this work.

\section{References}

1. Bergers G, Benjamin LE. Tumorigenesis and the angiogenic switch. Nat Rev Cancer. 2003;3(6):401-410.

2. Hood JD, Cheresh DA. Role of integrins in cell invasion and migration. Nat Rev Cancer. 2002;2(2):91-100.

3. Morales-Avila E, Ferro-Flores G, Ocampo-García BE, et al. Multimeric system of 99 mTc-labeled gold nanoparticles conjugated to c[RGDfK(C)] for molecular imaging of tumor alpha(v)beta(3) expression. Bioconjug Chem. 2011;22(5):913-922.

4. Xie H, Diagaradjane P, Deorukhkar AA, et al. Integrin alphavbeta3targeted gold nanoshells augment tumor vasculature-specific imaging and therapy. Int J Nanomedicine. 2011;6:259-269.

5. Lanzardo S, Conti L, Brioschi C, et al. A new optical imaging probe targeting alphaVbeta3 integrin in glioblastoma xenografts. Contrast Media Mol Imaging. 2011;6(6):449-458.

6. Streeter JE, Gessner RC, Tsuruta J, Feingold S, Dayton PA. Assessment of molecular imaging of angiogenesis with three-dimensional ultrasonography. Mol Imaging. 2011;10(6):460-468.

7. Bulte JW, Kraitchman DL. Iron oxide MR contrast agents for molecular and cellular imaging. NMR Biomed. 2004;17(7):484-499.
8. Wahajuddin, Arora S. Superparamagnetic iron oxide nanoparticles: magnetic nanoplatforms as drug carriers. Int J Nanomedicine. 2012;7: $3445-3471$.

9. Yu MK, Jeong YY, Park J, et al. Drug-loaded superparamagnetic iron oxide nanoparticles for combined cancer imaging and therapy in vivo. Angew Chem Int Ed Engl. 2008;47(29):5362-5365.

10. Gupta AK, Gupta M. Synthesis and surface engineering of iron oxide nanoparticles for biomedical applications. Biomaterials. 2005;26(18): 3995-4021.

11. Torchilin VP. Recent advances with liposomes as pharmaceutical carriers. Nat Rev Drug Discov. 2005;4(2):145-160.

12. Mulder WJ, Strijkers GJ, van Tilborg GA, Griffioen AW, Nicolay K. Lipid-based nanoparticles for contrast-enhanced MRI and molecular imaging. NMR Biomed. 2006;19(1):142-164.

13. Paganelli G, Magnani P, Zito F, et al. Pre-targeted immunodetection in glioma patients: tumour localization and single-photon emission tomography imaging of [99 mTc]PnAO-biotin. Eur J Nucl Med. 1994;21(4): 314-321.

14. Paganelli G, Magnani P, Zito F, et al. Three-step monoclonal antibody tumor targeting in carcinoembryonic antigen-positive patients. Cancer Res. 1991;51(21):5960-5966.

15. Diamandis EP, Christopoulos TK. The biotin-(strept)avidin system: principles and applications in biotechnology. Clin Chem. 1991;37(5): 625-636.

16. De Cuyper M, Joniau M. Magnetoliposomes. Formation and structural characterization. Eur Biophys J. 1988;15(5):311-319.

17. Boles KS, Schmieder AH, Koch AW, et al. MR angiogenesis imaging with Robo4- vs alphaVbeta3-targeted nanoparticles in a B16/F10 mouse melanoma model. FASEB J. 2010;24(11):4262-4270.

18. van Tilborg GA, Mulder WJ, van der Schaft DW, et al. Improved magnetic resonance molecular imaging of tumor angiogenesis by avidin-induced clearance of nonbound bimodal liposomes. Neoplasia. 2008;10(12):1459-1469.

19. Li W, Su B, Meng S, et al. RGD-targeted paramagnetic liposomes for early detection of tumor: in vitro and in vivo studies. Eur J Radiol. 2011;80(2):598-606.

20. Bulte JW, de Cuyper M, Despres D, Frank JA. Short- vs long-circulating magnetoliposomes as bone marrow-seeking MR contrast agents. J Magn Reson Imaging. 1999;9(2):329-335.

21. Efremova NV, Bondurant B, O'Brien DF, Leckband DE. Measurements of interbilayer forces and protein adsorption on uncharged lipid bilayers displaying poly(ethylene glycol) chains. Biochemistry. 2000;39(12): 3441-3451.

22. Ding N, Lu Y, Lee RJ, et al. Folate receptor-targeted fluorescent paramagnetic bimodal liposomes for tumor imaging. Int $J$ Nanomedicine. 2011;6:2513-2520.

23. Sipkins DA, Cheresh DA, Kazemi MR, Nevin LM, Bednarski MD, Li KC. Detection of tumor angiogenesis in vivo by alphaVbeta3-targeted magnetic resonance imaging. Nat Med. 1998;4(5):623-626.

24. Hsieh WJ, Liang CJ, Chieh JJ, et al. In vivo tumor targeting and imaging with anti-vascular endothelial growth factor antibody-conjugated dextran-coated iron oxide nanoparticles. Int J Nanomedicine. 2012;7:2833-2842.

25. Qiao J, Li S, Wei L, et al. HER2 targeted molecular MR imaging using a de novo designed protein contrast agent. PLoS One. 2011;6(3): e18103.

26. Schmieder AH, Caruthers SD, Zhang H, et al. Three-dimensional MR mapping of angiogenesis with alpha5beta1(alpha nu beta3)-targeted theranostic nanoparticles in the MDA-MB-435 xenograft mouse model. FASEB J. 2008;22(12):4179-4189.

27. Esposito G, Geninatti Crich S, Aime S. Efficient cellular labeling by CD44 receptor-mediated uptake of cationic liposomes functionalized with hyaluronic acid and loaded with MRI contrast agents. Chem Med Chem. 2008;3(12):1858-1862.

28. Sharkey RM, Karacay H, Cardillo TM, et al. Improving the delivery of radionuclides for imaging and therapy of cancer using pretargeting methods. Clin Cancer Res. 2005;11(19 Pt 2):7109s-7121s. 
29. Goodwin DA, Meares CF. Pretargeting: general principles; October 10-12, 1996. Cancer. 1997;80(Suppl 12):2675-2680.

30. Zhu W, Okollie B, Bhujwalla ZM, Artemov D. PAMAM dendrimerbased contrast agents for MR imaging of Her-2/neu receptors by a threestep pretargeting approach. Magn Reson Med. 2008;59(4):679-685.

31. Frascione D, Diwoky C, Almer G, et al. Ultrasmall superparamagnetic iron oxide (USPIO)-based liposomes as magnetic resonance imaging probes. Int J Nanomedicine. 2012;7:2349-2359.

32. Chen W, Jarzyna PA, van Tilborg GA, et al. RGD peptide functionalized and reconstituted high-density lipoprotein nanoparticles as a versatile and multimodal tumor targeting molecular imaging probe. FASEB $J$. 2010;24(6):1689-1699.
33. Mulder WJ, Strijkers GJ, Habets JW, et al. MR molecular imaging and fluorescence microscopy for identification of activated tumor endothelium using a bimodal lipidic nanoparticle. FASEB J. 2005;19(14): 2008-2010.

34. Kluza E, Jacobs I, Hectors SJ, et al. Dual-targeting of alphavbeta3 and galectin-1 improves the specificity of paramagnetic/fluorescent liposomes to tumor endothelium in vivo. J Control Release. 2012;158(2):207-214.
International Journal of Nanomedicine

\section{Publish your work in this journal}

The International Journal of Nanomedicine is an international, peerreviewed journal focusing on the application of nanotechnology in diagnostics, therapeutics, and drug delivery systems throughout the biomedical field. This journal is indexed on PubMed Central, MedLine, CAS, SciSearch ${ }^{\circledR}$, Current Contents ${ }^{\circledR} /$ Clinical Medicine,

\section{Dovepress}

Journal Citation Reports/Science Edition, EMBase, Scopus and the Elsevier Bibliographic databases. The manuscript management system is completely online and includes a very quick and fair peer-review system, which is all easy to use. Visit http://www.dovepress.com/ testimonials.php to read real quotes from published authors.

Submit your manuscript here: http://www.dovepress.com/international-journal-of-nanomedicine-journal 